\title{
Offset Compensation Method Using Photoelectric Switches for Positioning Control System ${ }^{\dagger}$
}

\author{
Naoyuki Tamaru*, Joji Yamaguchi*, Yasuhide Nishida* \\ and Fumikazu OHIRA*
}

\begin{abstract}
An offset compensation method using reflection-type photoelectric switches is studied with the aim of achieving a low-cost and highly accurate positioning control system. This system uses an open-loop control type robotic mechanism consisting of pulse motors and ball screws to reduce the cost. To make an optical connection in an automated optical cross-connect switching system, the robot must position optical fiber plugs at desired positions. The "on" region of the photoelectric switches is determined by scanning the plug grasped by the robot hand in front of the photoelectric switches. Offset compensation is achieved by calculating the difference between the initial position of the center of the region and the newly measured value. Experimental results show that the repetition error of the offset detection is less than $5 \mu \mathrm{m}$ (about $0.1 \%$ of the control position pitch) and the position variation of the center of the "on" region is less than $30 \mu \mathrm{m}$ when the distance between the switch and the plug is $0.1-1.0$ $\mathrm{mm}$. The feasibility of this compensation method using photoelectric switches was confirmed by installing it into the automated optical cross-connect switching system.
\end{abstract}

Key Words : photoelectric switch, offset compensation, robot, positioning control

\section{Introduction}

Recently a small, high-precision robotic mechanism has been made through mechatronics. It became possible mainly as a result of high-speed signal processing by microcomputers and high-performance actuators ${ }^{1,2)}$.

It is effective to use an open-loop control type robotic mechanism consisting of pulse motors and ball screws because of their low cost and small size. Therefore, we applied this mechanism to an automated optical crossconnect switching system ${ }^{3 / \sim 5}$, which cross-connects the selected optical-subscriber cable with the desired opticaloffice-equipment cable. The robot in the automated optical cross-connect switching system controls the connection/disconnection of the optical fibers as it inserts a plug with a fiber into the fiber adaptor on the adaptor alignment board or removes one from the adaptor. The problem is that it is difficult to move the robot hand to the target position precisely when thermal expansion occurs or the positions of the mechanical origins change, because the motor coordinates do not coincide with the target coordinates that were fixed in advance. If the ambient tempera-

†第 37 回自動制御連合講演会で発表 (1994・11)

* NTT Opto-electronics Laboratories, 3-9-11, Midori-cho, Musashino

(Received July 10, 1995)

(Revised December 24, 1996) ture varies by 30 degrees, the $250 \times 200 \mathrm{~mm}$ adaptor alignment board expands by about $0.1-0.2 \mathrm{~mm}$ in the case of non-uniform expansion between the robot mechanism and the adaptor alignment board. In the worst case the robot hand fails to reach the target position and cannot connect the fibers properly, because the target position is not sensed.

One proposal for decreasing the position error is to control the robot hand precisely by detecting the target position with a CCD (charge coupled device) sensor ${ }^{6)}$ or a PSD (position sensitive device) sensor ${ }^{7}$. However, it is difficult to locate the CCD or PSD sensor at the small tip of the robot hand. Moreover, the sensor and signal processing circuits for it are expensive.

The other proposal ${ }^{8)}$ is to detect the position of the robot hand relative to the target positions (i. e. shelves in the optical disk cartridge storehouse) using photoelectric switches in the robot hand and reflection marks formed on the front surface of each target. However, this system cannot compensate for the mechanical variation from the photoelectric switches to the optical disk cartridge being grasped by the hand, and the robot hand becomes larger and more complicated mechanism because of installing photoelectric switches.

In order to obtain a low-cost, highly accurate positioning control for an optical fiber handling robot, we studied an offset compensation method using simple and small photoelectric switches. The photoelectric switches are 
installed in an adaptor alignment board. The positions of the photoelectric switches are determined by scanning the plug grasped by the robot hand in front of the photoelectric switches. This paper describes the principle of this method, presents results of measuring the characteristics, and shows the effectiveness of adopting this method to the automated optical cross-connect switching system.

\section{Mechanical Configuration of the Robot}

Figure 1 shows the configuration of a positioning system for a fiber handling robot and the arrangement of photoelectric switches in an adaptor alignment board. The robot consists of a hand, three pulse motors, and three-axis mechanical guides with ball screws. The robot controls the connection and disconnection of the optical fibers by inserting a plug with a fiber into the fiber adaptor on the adaptor alignment board or by removing one from the adaptor. In this plug connection/disconnection procedure the robot hand is controlled by the $x, y$, and $z$-axis pulse motors with an open-loop (without feeding back the photoelectric switch signals). A ferrule is set on the tip of the plug, and there is a hole for the ferrule in the center of the adaptor. The plug with a fiber is connected to the adaptor by inserting the ferrule of the plug into the hole in the adaptor.

Figure 2 shows the arrangement of photoelectric switches and the adaptor alignment board. The adaptor alignment board contains many small holes for the

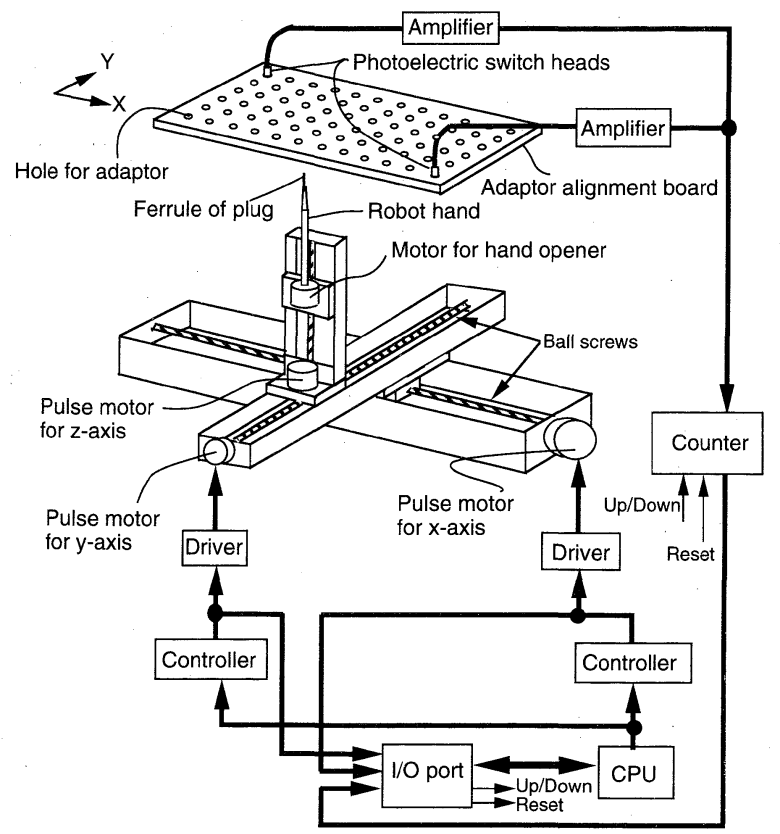

Fig. 1 Configuration of positioning system for a fiber handling robot and arrangement of photoelectric switches adaptors in a precise regular pattern. Two reflection-type photoelectric switches are buried in the holes set at diagonally opposite corners on the adaptor alignment board.

\section{Compensation Method}

Once the positions of the two holes at diagonally opposite corners on the adaptor alignment board have been measured using photoelectric switches, as described in section 3.1, all the other hole positions in the adaptor alignment board can be calculated by interpolation with the assumption that a temperature change causes linear and isotropic movement of the adaptor alignment board.

\section{1 Detection of hole center}

The reflection-type photoelectric switch can detect light reflected from a target object and outputs an "on/off" signal according to the reflected power. That is to say, the photoelectric switch outputs the "on" signal when the target object approaches it and the power entering the detection fiber exceeds the threshold level for an "on" state. In our experiment, the target object was a ferrule, which is the tip of an optical plug.

In the offset compensation procedure the robot hand grasping the plug with the ferrule approaches the photoelectric switch head to within about $1 \mathrm{~mm}$ and scans the plug by small step movements along the $x$ and $y$-axes in the plane perpendicular to the hole of the adaptor. The robot detects two transition positions as the signal changes from "off" to "on" and from "on" to "off" when the robot scans along each axis. The center of the "on" region is calculated as the position midway between the two transition points at each scan.

Figure 3 shows the calculation method for detecting the "on" region of the photoelectric switch. The circle is the

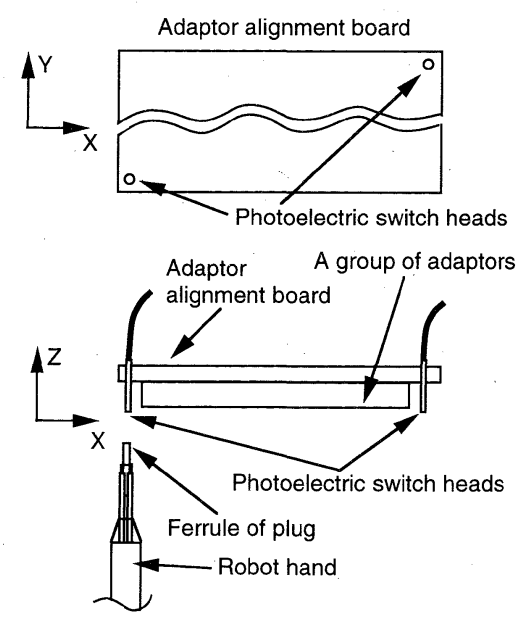

Fig. 2 Arrangement of photoelectric switches and an adaptor alignment board 


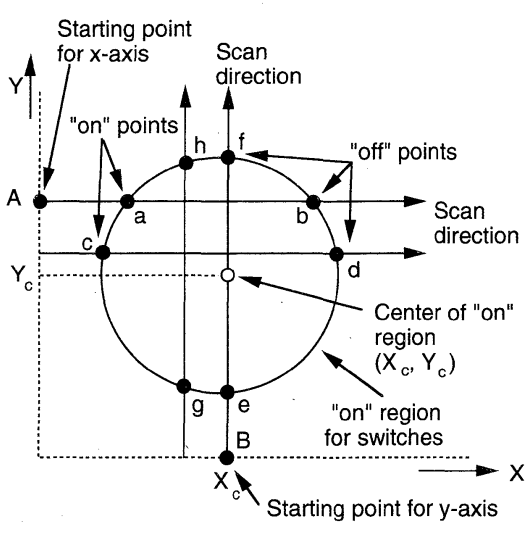

Fig. 3 Calculation method for detecting "on" region

border that separates the "on" region from the "off" region.

First, according to the internal counter of the CPU, the robot grasping the ferrule of the plug positions the hand at point $A$, i. e. the starting point for $x$-axis scanning. The internal counter, which has more than 24 bits, is composed of the IC memory (RAM) of the CPU and is controlled by the robot movement program. Point $A$ is located slightly before the "on" region considering the maximum change in size of the adaptor alignment board with change in temperature. Because the maximum size change is assumed to be 0.1-0.2 mm, point $A$ should be set more than $0.6 \mathrm{~mm}$ ("on" region radius $0.4 \mathrm{~mm}+0.2 \mathrm{~mm}$ ) away from the previously preset center point of the photoelectric switch. The robot scans in the positive direction of the $x$-axis step by step with the $x$-axis pulse motor, counts the number of steps as pulse number, and checks the state of the output signal of the photoelectric switch to see whether the signal is "on". The point $a$ is measured using the unit of the step pulse number of the $x$-axis pulse motor from the starting point $A$ for the $x$-axis. If the signal is "off", the robot continues to scan another step until the signal is "on". When the signal becomes "on" at point $a$, the pulse number from the starting point $A$ to point $a$ is sent to the CPU and stored.

The robot continues to scan along the $x$-axis until the state of the signal is "off". When the signal becomes "off" at point $b$, the pulse number from the starting point $A$ to point $b$ is sent to the CPU and stored. The detection precision can be improved by making several scans at different places for each axis. Similarly, the points $e$ to $h$ are measured using the pulse number of the $y$-axis pulse motor.

By using the internal counter the robot controls the movement of its hand to the starting point for the other switch that is located at the diagonally opposite corner on the adaptor alignment board as in the former method. The

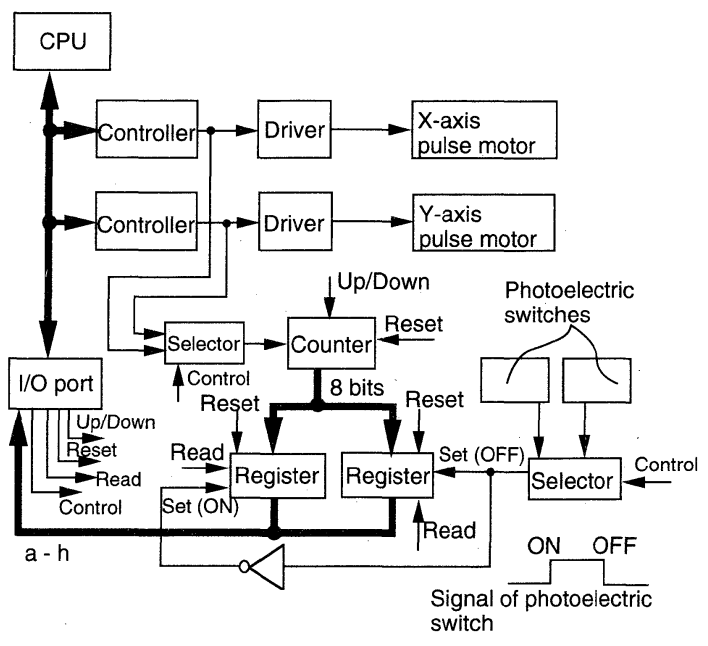

Fig. 4 Block diagram of offset detection circuit

starting point is determined by the preset center position renewed by the latest offset measurement results and also scans along the $x$-axis and the $y$-axis.

In this figure the center point $X_{c}$ for the $x$-axis is calculated from

$$
X_{c}=(a+b+c+d) / 4,
$$

and the center point $Y_{c}$ for the $y$-axis is similarly

$$
Y_{c}=(e+f+g+h) / 4 \text {. }
$$

The center point of the "on" region is determined by measuring at least three transition points because a circle is defined by three points. We actually made eight measurements in order to get high precision(i. e. less than 20 $\mu \mathrm{m})$.

If in advance, the real hole center and the center of the "on" region are measured at the same time, the new hole center shifted by temperature change can be calculated from the new measurement of the center of the "on" region by using this relation. In this calculation it is assumed that the change in size of the adaptor alignment board is linear and isotropic due to expansion, rotation and displacement.

It is necessary to use this offset compensation method when the plug connection/disconnection operation fails due to positioning error of the robot hand or when the mechanical components of the robot are renewed. Therefore, in the case of the usual plug connection/disconnection operation, it is not necessary to use the offset compensation procedure, but the robot controls the hand according to the latest offset results achieved.

\subsection{Detection circuit}

Figure 4 shows the block diagram of the offset detection circuit. The robot hand is controlled by the $x$-axis pulse motor and the $y$-axis pulse motor. The motor driving pulses from the controllers are selected and counted. The 


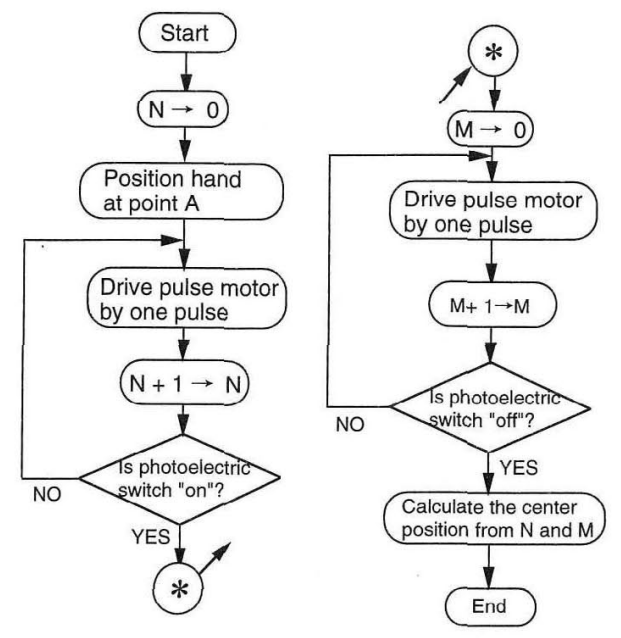

Fig. 5 Flow chart for detecting the center of the "on" region

pulse counts are stored in two registers, one for the "on" point, and the other for the "off" point. The counter and the registers have 8-bit resolution. Therefore, the scanning distance of the robot hand for the offset measurement is about $2.5 \mathrm{~mm}$, because the step distance of the pulse motor is $10 \mu \mathrm{m}$. The scanning distance is sufficient when it is longer than $2 \mathrm{~mm}$, because the diameter of the "on" region is about $0.8 \mathrm{~mm}$. The data stored in the registers is transferred to the CPU through the $\mathrm{I} / \mathrm{O}$ port, and the control signals for the counter, selectors, etc. are output by the CPU through the I/O port.

\section{3 Flow chart of measurement procedure}

Figure 5 shows the flow chart for detecting the center point of the "on" region. In this flow it is not necessary to use a counter and register circuit as shown in Fig. 4. This procedure reduces the hardware cost, but the measurement time of this procedure becomes longer than that of the method in Fig. 4. The CPU checks whether the state of the photoelectric switch is "on" or "off". If the state is different from that in the previous step, the step numbers $N$ and $M$ from the initial point $A$ to the transition position are recorded in the CPU. The center point of the "on" region for the $x$-axis is calculated from the numbers $N$ and $M$. Similarly, the center point for the $y$-axis is calculated by scanning the hand along the $y$-axis.

\section{Characteristics of "on" Region}

\section{1 Experimental setup}

Figure 6 shows the experimental arrangement for measuring the switching characteristics. The top surface of the ferrule for the optical plug was used as the reflection object for the photoelectric switches. The plug is consisted of the cylindrical ferrule and the metallic holder that supports the root of the ferrule. The plug was an MU-type

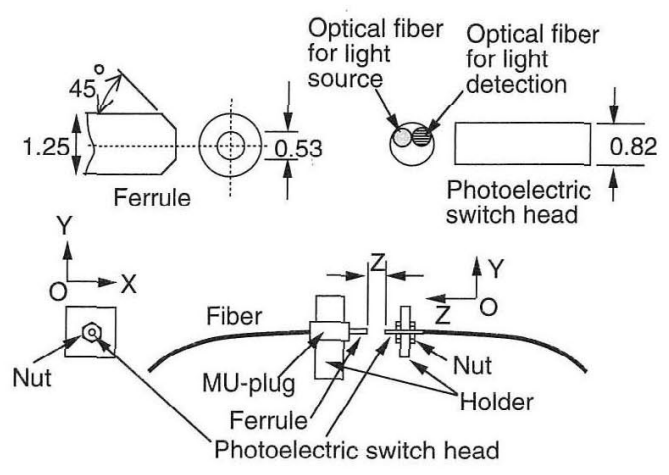

Fig. 6 Experimental arrangement for measuring switching characteristics

Table 1 Specifications of a photoelectric switch and a target object

\begin{tabular}{|c|c|c|}
\hline \multirow{5}{*}{$\begin{array}{l}\text { Photoelectric } \\
\text { switch }\end{array}$} & Detection type & $\begin{array}{l}\text { Reflective type } \\
\text { (not through-beam } \\
\text { type) }\end{array}$ \\
\hline & Light source & $\begin{array}{l}\text { Red LED light } \\
(660 \mathrm{~nm})\end{array}$ \\
\hline & Output response time & $250 \mu \mathrm{s}$ \\
\hline & $\begin{array}{l}\text { Maximum detection dis- } \\
\text { tance for } 10 \mathrm{~mm} \times 10 \mathrm{~mm} \\
\text { white paper }\end{array}$ & $4 \mathrm{~mm}$ \\
\hline & Smallest detectable object & $15 \mu \mathrm{m} \phi$ copper wire \\
\hline \multirow{2}{*}{ Target object } & Object & $\begin{array}{l}\text { Top surface of } \\
\text { ferrule }\end{array}$ \\
\hline & Reflection surface & $\begin{array}{l}\text { Polished surface of } \\
0.5 \mathrm{~mm} \phi\end{array}$ \\
\hline
\end{tabular}

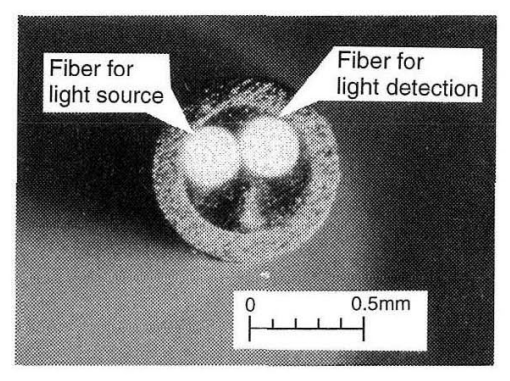

Photo. 1 Microscopic photograph of a photoelectric switch head

optical connector with a ferrule $1.25 \mathrm{~mm}$ in diameter. The surface of the ferrule that is made of ceramics, was polished precisely.

The plug was fixed on a three-axis movable table and located in front of the photoelectric switch. We measured the "on" and "off" positions of the photoelectric switch by moving the plug. The measurement precision was $10 \mu \mathrm{m}$. Table 1 shows specifications of the photoelectric switch and the target object. Photo. 1 shows the photoelectric switch head containing two fibers, one for the light source, 
the other for the light detection. The red light of $660 \mathrm{~nm}$ is emitted from the light source fiber installed in the head of the photoelectric switch and is reflected from the plug surface. The photoelectric switch head is connected to the switch amplifier by an optical cable.

\section{2 Experimental results}

\section{(1) “on" region}

Figures 7, 8 and $\mathbf{9}$ show measurement results for the "on" region when the distance $Z$ between the ferrule of the plug and the photoelectric switch was changed from $0.1 \mathrm{~mm}$ to $0.8 \mathrm{~mm}$. The amplifier gain $G$ of the photoelectric switch for Fig. 7 is 0.75 , for Fig. 8 is 1.25 and that for Fig. 9 is 1.75. Symbols such as “\#”, " $\$$ ” etc. represent measured values of "on" or "off" transition positions and circles show the trend in the measured values in these figures. The gain $G$ is shown as the rotation number of the variable resistance for the amplifier of the photoelectric switch. When the gain $G$ increases, the photoelectric

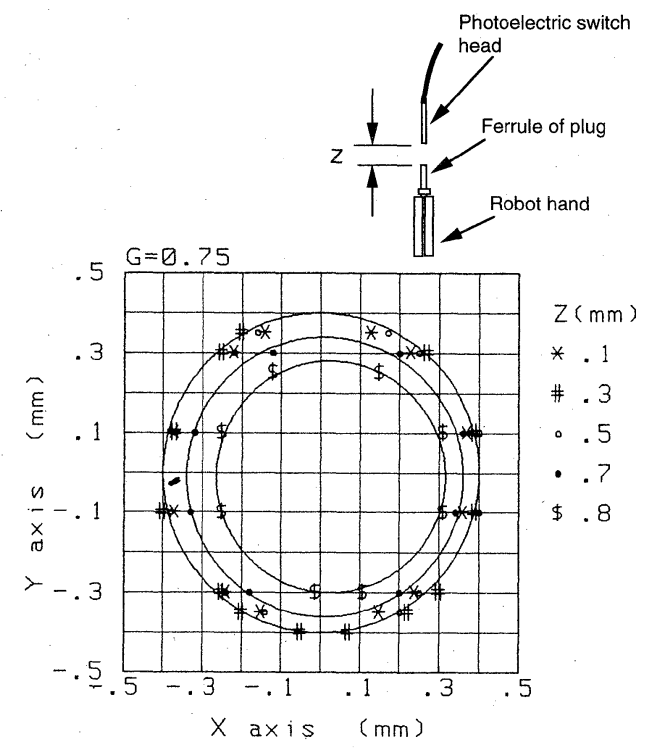

Fig. 7 Measurement results for "on" region at $G=0.75$

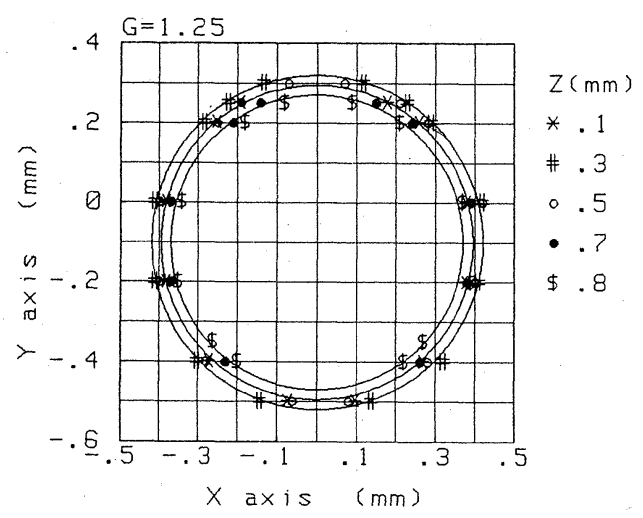

Fig. 8 Measurement results for "on" region at $G=1.25$ switch outputs the "on" state even when the reflected light power the weaker. That is to say, the threshold level for the "on" state of the photoelectric switch decreases for higher gain of the amplifier. In Fig. 7, the outermost circle is for $Z=0.3 \mathrm{~mm}$, the middle one is for $0.7 \mathrm{~mm}$, and the innermost one is for $0.8 \mathrm{~mm}$. In Fig. 8 , the outermost circle is for $Z=0.3 \mathrm{~mm}$, the middle one is for $0.1 \mathrm{~mm}$, and the innermost one is for $0.8 \mathrm{~mm}$. In Fig. 9 , the outermost circle is for $Z=0.3 \mathrm{~mm}$, the middle one is for $0.9 \mathrm{~mm}$, and the innermost one is for $1.1 \mathrm{~mm}$.

Figures $7-9$ show that the diameter of the "on" region is about $0.8 \mathrm{~mm}$. Therefore, it is effective if the robot hand scans at least $2 \mathrm{~mm}$ around the center of the photoelectric switch for the offset measurement. Moreover, they show that the measured values almost coincide with the exact circular lines. Therefore, we estimate that the light beam emitted from the photoelectric switch is also circular. Figure 7 indicates that the circle enclosing the "on" region decreased in diameter and the center of the "on" region shifted right and down when the distance $Z$ increased. But when the gain $G$ is 1.25 the center of the "on" region hardly shifts (less than $\pm 20 \mu \mathrm{m}$ ) from $Z=0.1 \mathrm{~mm}$ to 0.8 $\mathrm{mm}$. Even if the diameter of the "on" region changes, the center position of the "on" region will not change, when there is no shift of the "on" region. This phenomenon increases the detection margin. When the gain $G$ is 1.75 the variation in the detection of the center position of the "on" region is about $30 \mu \mathrm{m}$ from $Z=0.1 \mathrm{~mm}$ to $1.0 \mathrm{~mm}$.

(2) Detection margin

There is a variation in the distance between the photoelectric switch and the ferrule of the plug because of the positioning error of the robot and expansion of adaptor alignment board due to temperature change, when the robot moves the plug in front of the photoelectric switch. Therefore a wide detection margin is useful.

Figure $\mathbf{1 0}$ shows the detection margin for the distance

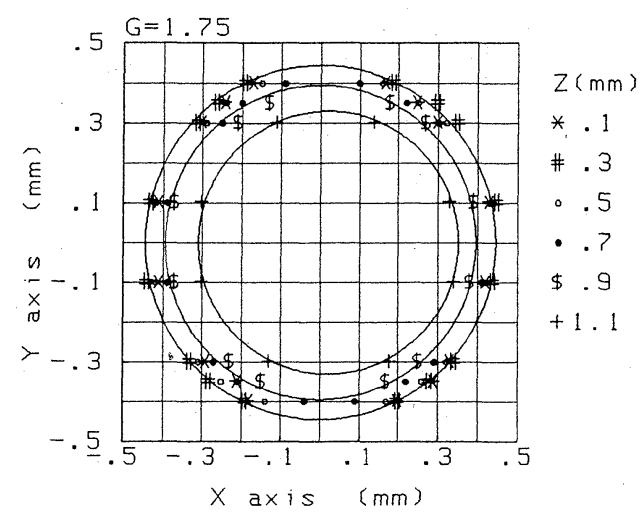

Fig. 9 Measurement results for "on" region at $G=1.75$ 


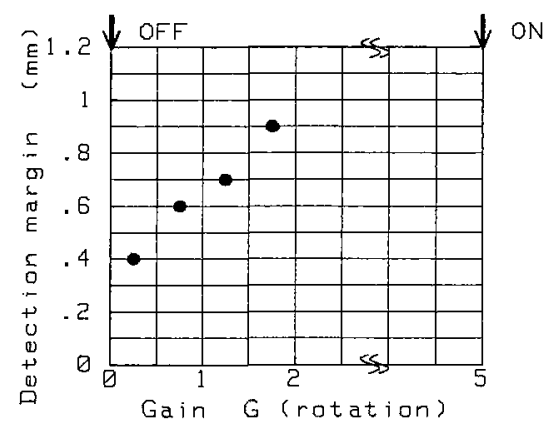

Fig. 10 Detection margin for distance along $z$-axis

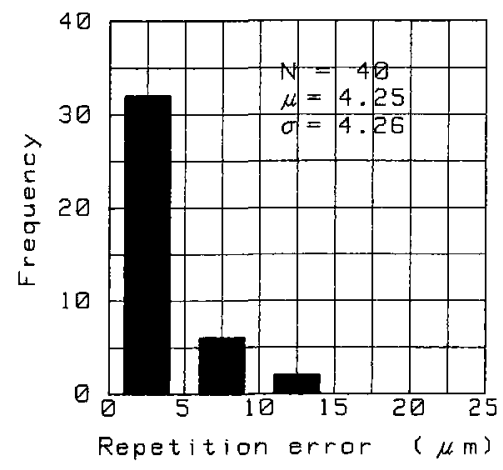

Fig. 11 Histogram of repetition error for offset measurement

along the $z$-axis versus amplifier gain $G$. The gain $G$ is set to 0 when the photoelectric switch just changes to the "off" state from the "on" state at the target position of the nominal distance $(Z=0.6 \mathrm{~mm})$ with gradually decreasing gain. When the gain $G$ is 5 , the photoelectric switch just changes to the "on" state even if there is no target near the switch. This figure shows that the detection margin increases with gain of the photoelectric switch. For $G=$ 1.75 , the detection margin is about $0.9 \mathrm{~mm}$.

(3) Detection error

We measured the detection error as variation of the offset measurement values for the same experimental conditions. That is to say, the detection error represents the repetition error. Figure $\mathbf{1 1}$ shows a histogram of the repetition error in the measurements made by the crossconnect switching robot that can jumper optical fibers. The robot controls the hand by $10-\mu \mathrm{m}$ steps and makes two scans in the same direction for each axis in order to reduce the measurement error. The two scans are about $0.1 \mathrm{~mm}$ apart.

The $x \cdot y \cdot z$ table is supported by linear guides and ball screws as shown in Fig. 1. The parallelism error of the linear guides for the $x, y$, and $z$ axes is less than $3 \mu \mathrm{m}$, the axial spacing of the ball screws for each axis is less than $5 \mu \mathrm{m}$, and the lead pitch error is less than $10 \mu \mathrm{m}$ for the full stroke.

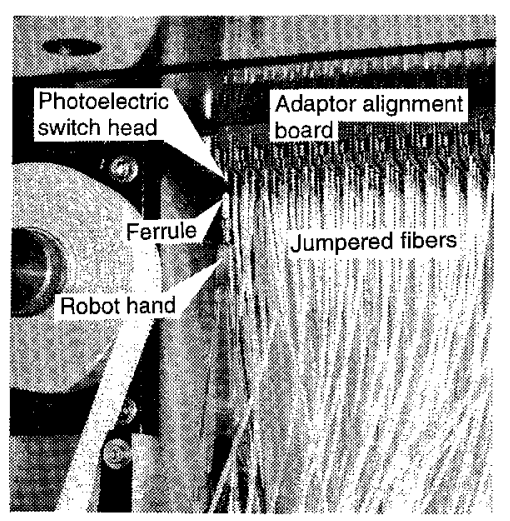

Photo. 2 Arrangement of a photoelectric switch head installed in the automated optical cross-connect switching system

In Fig. 11 the horizontal axis shows the repetition error of the offset detection and the vertical axis shows the frequency of the error. The total number of measurements was 40 , the average repetition error of the offset detection was about $4.3 \mu \mathrm{m}$ (about $0.1 \%$ of the control pitch), and the standard deviation was $4.3 \mu \mathrm{m}$. Therefore, the positioning error of all adaptors in the adaptor alignment board is considered to be below the target error of $20 \mu \mathrm{m}$ because the table precision error is less than a few micrometers.

(4) Effectiveness for automated optical cross-connect switching system

Photo. 2 shows the arrangement of a photoelectric switch head installed in an automated optical cross-connect switching system with 2000 adaptors and 1000 plugs. The adaptors are located in a rectangular lattice of $4 \times 6 \mathrm{~mm}$ on the adaptor alignment board. The compensation method as shown in Fig. 5 is used in the automated optical crossconnect switching system. It took about 70 seconds to finish the compensation procedure with two scans in the same direction for each axis at a pair of diagonal measurement points including the travel time to the measurement points from the origin point and the rise and fall time of the robot hand. That is to say, a total of eight measurements of "on" to "off" or "off" to "on" transition points were accomplished. This compensation method was very effective when the robot hand was changed because there was no need to recheck or remeasure the reference points precisely. Moreover, a fiber handling robot using this offset compensation method has operated for about one year without any problems.

This offset compensation system cannot be adapted only to the pulse motor control system but also to an $\mathrm{AC}$ servo motor control system that feeds back the motor encoder 
signal to the motor.

\section{Conclusions}

Offset compensation using reflection-type photoelectric switches should lead to a low-cost, highly accurate positioning control system. This system is necessary for a robotic mechanism that must position many identical objects such as electrical parts at desired positions.

The robot hand grasping an object (a plug for an optical fiber) scans in front of photoelectric switches and determines the "on" region. The center of the region is determined from the "on" region of the photoelectric switches. Offset compensation can be achieved by calculating the difference between the initial position of the center of the region and the newly measured value.

The experimental results show that the repetition error of the offset detection is less than $5 \mu \mathrm{m}$ (about $0.1 \%$ of the control position pitch) and the position variation for the center of the "on" region is less than $30 \mu \mathrm{m}$ when the distance between the photoelectric switch head and the ferrule of the plug is $0.1 \mathrm{~mm}$ to $1.0 \mathrm{~mm}$. The effectiveness of the compensation method using photoelectric switches was confirmed by installing it into an automated optical cross-connect switching system and controlling the robot to exchange plugs.

\section{Acknowledgments}

The authors would like to thank Tsuneo Kanai, Kazumasa Kaneko and Tetsufumi Shoji for their valuable discussions.

\section{References}

1) S. Hosokawa, A. Nagayama, and K. Nishimura: Advanced Tape Cartridge Handling Techniques for Automated MSS's, IEEE Trans. on Magnetics, 27-5, 4382/ 4387 (1991)

2) M. Sakai, A. Nagayama, and K. Sasakura: Positioning Method Using Polarization Optical Sensor for Precision Robot Systems, Proc. of SPIE, Vol, 1385, Optics Illumination, and Image Sensing for Machine Vision V, 8/14 (1990)

3) N. Tamaru, F. Ohira, Y. Nishida, T. Kanai, T. Shoji, J. Yamaguchi and H. Kimura: Automated Optical MainDistributing-Frame by Fiber Handling Robot, 1994 Autumn national convention record, IEICE, B-838, 436(1994) in Japanese

4) Y. Nishida, F. Ohira, T. Kanai, N. Tamaru, T. Shoji, J. Yamaguchi and $\mathrm{H}$. Kimura: Main Distributing Frame Using a Fiber-handling Method in Optical Access Networks, Optical Fiber Conference(OFC)'95 Technical Digest, TuK 6, 57/58(1995)

5) H. Kimura, Y. Nishida, F. Ohira and S. Umemura: Optical Fiber Handling System Design for Automated Optical Main Distributing Frame(MDF), 1994 Autumn national convention record, IEICE, B-839, 437 (1994) in Japanese

6) N. Tamaru, M. Makihara, S. Inagaki, A. Nagayama, and
K. Sasakura: Supply and Removal of Small Amounts of Liquid Using a Robot Mechanism, JSME International Journal, Series C, 38-2, 300/306 (1995)

7) K. Sasakura, A. Nagayama, and M. Sakai: New Polarization-detecting Optical Sensor for Precision Robot System, Technical digest of conference on Lasers and Electro-optics (CLEO), Vol. 7, CFS 2, 564/565(1990)

8) K. Kaneko, M. Takayanagi, I. Yamada and K. Katoh: High-speed Media Handling Technology for Optical Mass Storage System, NTT R\&D, 41-3, 353/362(1992) in Japanese

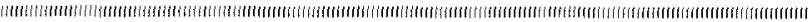

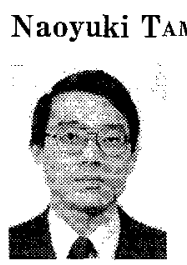

(Member)

He received the B.S., M.S. and Dr. degrees in electrical engineering from $W$ aseda University, Tokyo, Japan, in 1970, 1972, and 1992 respectively.

Since joining NTT in 1972 , he has been engaged in research and development on the positioning control for file memory systems and for optical switching equipments.

$\mathrm{He}$ is a member of the Institute of Electronics, Information and Communication Engineers of Japan, and the IEEE.

Joji Yamaguchi

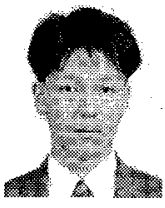

He received B.E., M.E. and Dr. degrees in mechanical engineering, all from Tokyo Institute of Technology, Tokyo, Japan, in 1988, 1990 and 1993 , respectively.

He joined NTT Interdisciplinary Research Labs., Tokyo, Japan, where he is engaged in research on optical cross-connect systems. Dr. Yamaguchi is a member of the Japan Society of Mechanical Engineers, and the Japan Society for Precision Engineering.

\section{Yasuhide Nishida}

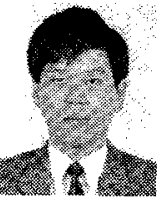

He received the $B . E$. and $M . E$. degrees in mechanical engineering from Tohoku Univer sity, Sendai, Japan in 1976 and 1978, respectively.

He joined NTT Electrical Communication Laboratories in 1978 and since then he has been engaged in research and development on mass storage systems and telecommunication mechatronics systems. He is now a Senior Research engineer, Supervisor at Optoelectronics Laboratories. Mr. Nishida is a member of the Japan Society of Mechanical Engineers, the Japan Society of Applied physics and Optical Society of America. 
Fumikazu OHira

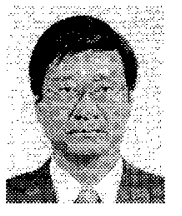

He received the B.E., M.E. and Ph. D degrees in precision engineering from Osaka University, Osaka, Japan, in 1973, 1975, and 1989, respectively.

In 1975, he joined NTT, where he has been engaged in research and development of precisionmachining and packaging technologies of electronic and optical components. Dr. Ohira is a member of the Institute of Electronics, Information and Communication Engineers of Japan, and the Japan Society for Precision Engineering. 